\title{
OBSERVATIONS OF THE POLAR MAGNETIC FIELDS
}

\author{
JAN OLOF STENFLO
}

Astronomical Observatory, Lund, Sweden

\begin{abstract}
Results from observations of the polar magnetic fields made in 1968 with the Mount Wilson magnetograph are reported. The field was of south polarity near the heliographic north pole and of north polarity near the south pole. The inversion line of the field was at latitude $+70^{\circ}$ in the north hemisphere and $-55^{\circ}$ in the south, which coincides with the position of the polar prominence zones at that time. Observations made simultaneously at the Crimean Astrophysical Observatory are in good agreement with the Mt. Wilson data and confirm the latitude variation of the field.

A few hundred of Hale's plates from 1914, which he had used to determine the Sun's general magnetic field, have been remeasured. The plates were automatically scanned with a digitized microphotometer, and the treatment of the data was made with a computer. While the visual measurements by Van Maanen had given a strong general field similar to that of a dipole, the computer reductions did not reveal any significant field (stronger than $5 \mathrm{G}$ ) at any latitude.
\end{abstract}

\section{Introduction}

The various theories of the solar cycle make different predictions about the magnetic fields at high heliographic latitudes. Although the polar fields are so important for tests of the solar cycle theories, our knowledge of the behaviour of the polar fields is very limited. It is difficult to observe the polar fields because they are very weak and always situated close to the limb.

The first attempts to determine the average properties of the Sun's magnetic field were made by Hale (Hale, 1912, 1913; Hale et al., 1918; Seares et al., 1918, 1919). $\mathrm{He}$ found a latitude variation of the field between latitudes $-50^{\circ}$ and $+50^{\circ}$ that corresponded to that of a dipole-type field. The polarity of the solar magnetic field was the same as that of the Earth. Assuming a dipole-type field for the Sun Hale could calculate what the field strength would be at the poles. The observed field was however found to depend very much on the spectral line used; the field was stronger in weaker lines. This was interpreted in terms of a depth-dependence of the field. Accordingly the polar field strength would vary from about $50 \mathrm{G}$ in the lower photosphere to zero in the upper photosphere. This extremely rapid variation of the field with height is however quite inconsistent with the assumption of a dipole-type field and implies that the field lines should be almost parallel to the solar surface (Rosseland, 1925; Stenflo, 1970b).

Determinations of the inclination $i$ of the magnetic axis to the rotational axis and the period of rotation $P$ of the magnetic pole around the axis of rotation were also presented (Seares et al., 1918, 1919). The results were: $i=6^{\circ} .0 \pm 0.4$ and $P=31.52 \pm 0.28$ days. The magnetic pole was on the central meridian at time $t=\mathrm{June} 25.38 \pm 0.42$ (UT), 1914.

It has long been a riddle what these early determinations of the Sun's general magnetic field really mean, because later observations have given entirely different results. Hale tried to make a new determination of the Sun's general field in 1931-1932 
but obtained zero results. This made him suspect that the solar magnetic field could vary in time with an unknown period (Mount Wilson Observatory Year Book 33, 1933-1934). It is interesting to note that the determinations of the general magnetic field by Hale in 1912-1914 were made during a period of minimum solar activity, which was the lowest minimum we have had since 1810 . It was preceded by the lowest maximum there has been since 1816 .

The first extensive photoelectric observations of the fields at high heliographic latitudes were made by Babcock and Babcock (1955). The observed longitudinal field was of the order of $1 \mathrm{G}$ and showed opposite sign in the two hemispheres. The polarity of the field was opposite to that of the Earth. In the spring of 1957 the field near the south pole was observed to reverse its sign (Babcock, 1959). The field seemed to be of the same polarity (directed outwards) at both poles for $1 \frac{1}{2} \mathrm{yr}$, until it suddenly, in November 1958, reversed sign at the north pole. It was suggested by Waldmeier (1960) that this difference in time of the field reversals was related to the difference in phase between the activities in the two hemispheres.

On the basis of these observations of the reversal of the polar fields Babcock (1961) proposed a theory for the solar cycle according to which the field will reverse its sign around each period of maximum solar activity. This theory has been significantly developed by Leighton (1959), who however assumes infinite electrical conductivity and thereby avoids the problem of the maintenance of the field. Steenbeck and Krause $(1966 a, b, 1967,1969)$ succeeded to build up a solar cycle theory that is based on a dynamo mechanism for the maintenance of the field and which qualitatively yields the same results as the phenomenological theory by Babcock. All these theories predict a polarity of the solar field in the period 1912-1914 which is opposite to that found by Hale.

\section{Observations of the Polar Fields near the Last Maximum of Solar Activity}

As an actual reversal of the polar fields has only been observed once, in 1957-1958, it is of considerable interest to study the polar fields around the activity maximum in 1968-1969. I will here report on systematic observations that were carried out with the Mt Wilson magnetograph during July and August 1968 (Stenflo, 1970a). The north and south polar regions were scanned almost daily during a period covering approximately two solar rotations. The scanning aperture was $5^{\prime \prime} \times 5^{\prime \prime}$, and the spectral line Fer $5250.2 \AA$ was used.

A computer program was written to plot synoptic charts of the polar regions. Synoptic charts for August 1968 are presented in Figure 1. These maps show isogauss contours for $H_{\|} / \cos \theta$, where $H_{\|}$is the observed longitudinal field and $\theta$ the heliocentric angle. $H_{\|} / \cos \theta$ is the total field strength if the field is assumed to be directed along the solar radius. It is often quite misleading to present observations of polar magnetic fields in terms of $H_{\|}$only, since the observations are often made at various heliocentric angles. Close to the limb the magnetic field should be mainly transversal.

$\mathrm{N}$ polarity elements seem to predominate in both polar regions. Accordingly one 


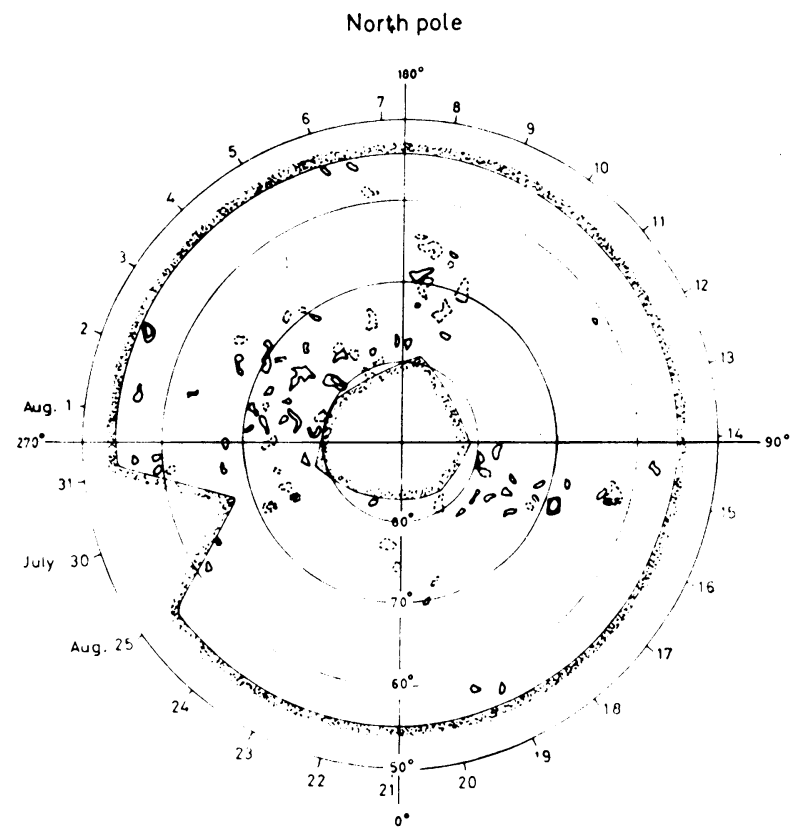

Fig. 1a.

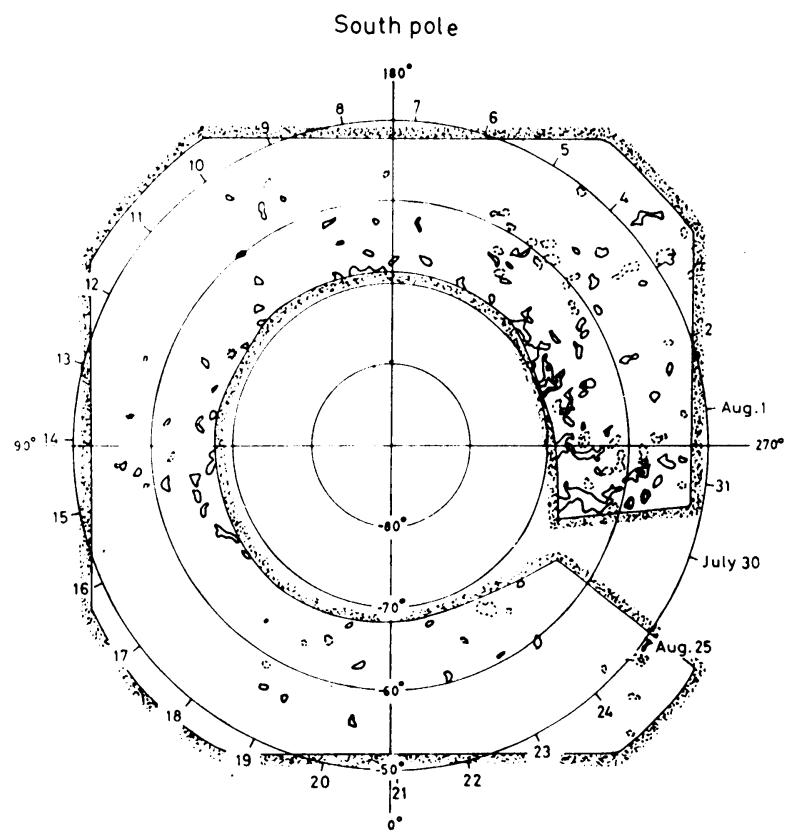

Fig. 1b.

Fig. 1a-b. Synoptic charts of the polar magnetic fields $H_{\|} / \cos \theta$. Solid lines represent $\mathrm{N}$ polarity, dashed lines $S$ polarity. The isogauss levels are 10 and $20 \mathrm{G}$. The areas covered by the observations are enclosed by curves with shadings on the outside. (a) North pole. Observations from August 1August 23, 1968. (b) South pole. Observations from July 31-August 23, 1968. 
could get the impression that the average magnetic field was directed outwards from both poles, a situation similar to that observed by Babcock (1959) for the field in 1957-1958. A more careful analysis shows however that this is not quite so.

The structure of the polar fields is very complicated and is far from rotationallysymmetric. Some average properties may be seen, however, by averaging the radial field $H_{\|} / \cos \theta$ over all longitudes and studying the latitude variation of this field. Figure 2 shows the latitude variation of the field for July-August 1968. $H$ is the observed average radial field multiplied by a factor of 3 to account for the weakening of the $5250 \AA$ line in magnetic regions (Harvey and Livingston, 1969). It is hoped that $H$ by this correction will be a good approximation of the true average field strength. The synoptic charts on the other hand have not been corrected for line weakenings.

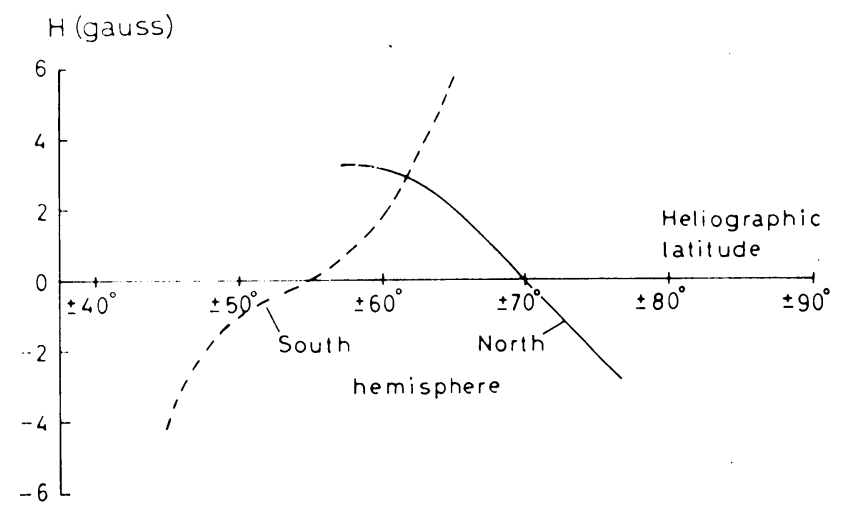

Fig. 2. The average radial magnetic field of July-August, 1968, corrected for line weakenings in magnetic regions.

The field in the north hemisphere varies in strength from about $+3 \mathrm{G}$ at lat. $+60^{\circ}$ to $-2 \mathrm{G}$ at $+75^{\circ}$, with polarity reversal at lat. $+70^{\circ}$. In the south hemisphere it varies from $-4 \mathrm{G}$ at lat. $-45^{\circ}$ to about $+6 \mathrm{G}$ at $-65^{\circ}$, with polarity reversal at lat. $-55^{\circ}$. The field is of $\mathrm{N}$ polarity in both polar regions between lat. $55^{\circ}$ and $70^{\circ}$. This is the reason why one gets the impression that the field is directed outwards from both poles.

The mean latitudes of the polar prominence zones in 1968 were $+72^{\circ}$ and $-58^{\circ}$ (Waldmeier, 1969). This coincides within the limits of observing accuracy with the latitudes of polarity reversals in Figure 2. Such a relation between inversion lines of the average magnetic field at high latitudes and the polar prominence zones was first suggested by Waldmeier (1960). It is also of interest to compare our result with that of Bumba et al. (1969) that quiescent prominences and filaments at high latitudes coincide in position with minima in the radio emission at $1420 \mathrm{MHz}$ and with minima in the brightness of the green $(\lambda 5303 \AA)$ corona.

The asymmetry or phase-shift between the two hemispheres may be explained in the theory of Steenbeck and Krause $(1966 \mathrm{a}, \mathrm{b}, 1967,1969)$ in terms of a constant 
quadrupole-type field that is superposed on a variable dipole-type field. When the dipole-type field disappears, only the quadrupole-type field, which has the same polarity at both poles, is seen.

\section{Comparison of Observations at Mt Wilson and Crimea}

It is important that such delicate observations as those of the polar fields can be verified by other observers, if possible by simultaneous observations. On August 17 and 20 the polar fields were recorded at Crimea and Mt Wilson with a time difference of only about $10 \mathrm{hr}$ between the recordings at the two observatories (Kotov and Stenflo, 1970). The position of the regions scanned is illustrated in Figure 3. The characteristics of

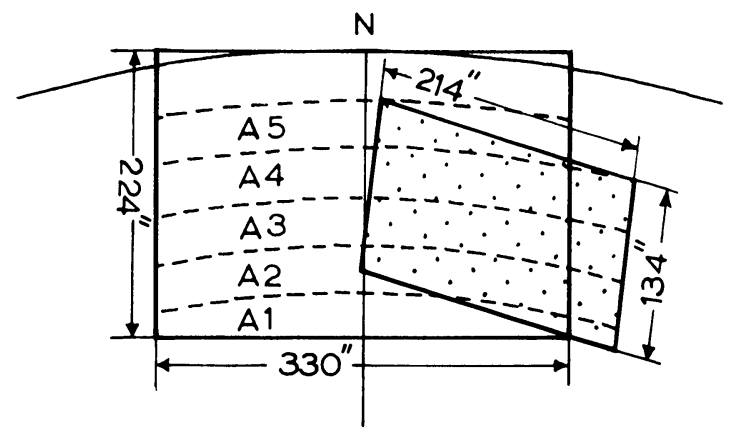

Fig. 3. Illustration of the north polar regions scanned at Mt Wilson and Crimea (with dots inside). For the reduction, the scanned regions were divided in smaller areas A1-A5, in which the average properties of the field were determined.

TABLE I

Data for the recordings with the Mt Wilson and Crimean magnetographs

\begin{tabular}{lll}
\hline Observatory & Mt Wilson & Crimea \\
\hline Scanned area & $330^{\prime \prime} \times 224^{\prime \prime}$ & $214^{\prime \prime} \times 134^{\prime \prime}$ \\
Slit size & $5^{\prime \prime} \times 5^{\prime \prime}$ & $9^{\prime \prime} \times 2^{\prime \prime} .5$ \\
Scanning velocity $v$ & $5^{\prime \prime} .9 / \mathrm{s}$ & $1^{\prime \prime} / \mathrm{s}$ \\
Time constant $\tau$ & $0^{8} .4$ & $2^{8} .5$ \\
R.m.s. noise & $3 \mathrm{G}$ & $1.7 \mathrm{G}$ \\
Position of exit slits & $7-87 \mathrm{~m} \AA$ & $27-90 \mathrm{~m} \AA$ \\
\hline
\end{tabular}

the two magnetographs are compared in Table I. In Figure 4 two isogauss maps covering the same region on the Sun are shown. The six largest magnetic elements in the Crimean map could all be identified in the Mt Wilson map. Due to the time difference the Crimean elements are displaced in the westward direction by about $6^{\circ}$ relative to the Mt Wilson elements. These magnetic features obviously have lifetimes exceeding $10 \mathrm{hr}$. The other isogauss maps do not show such a good agreement as the maps in Figure 4. 
N

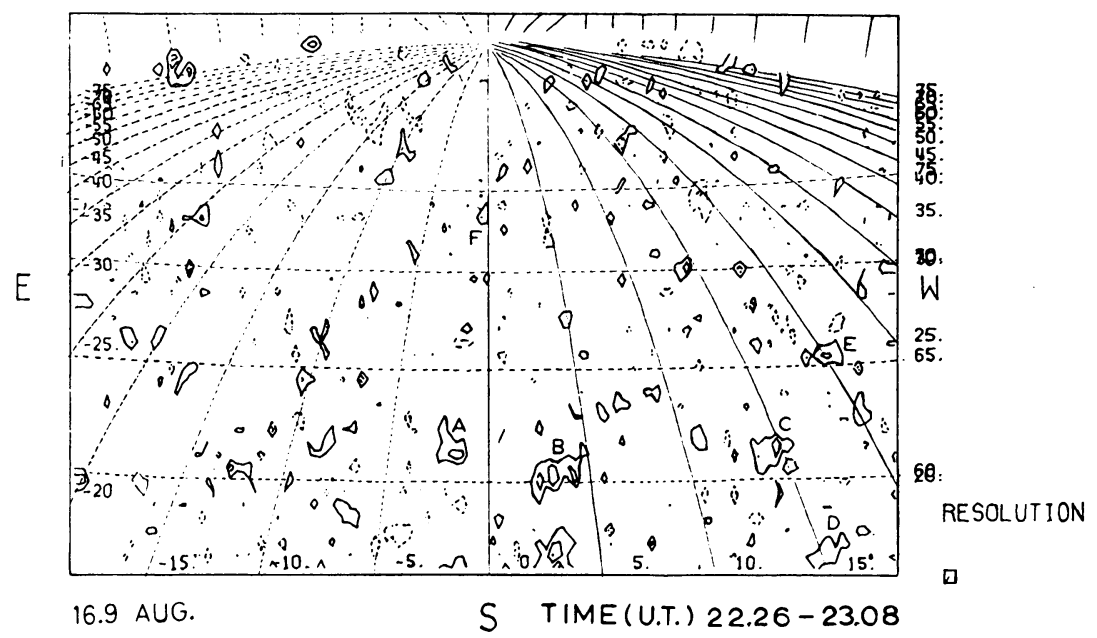

Fig. 4a.

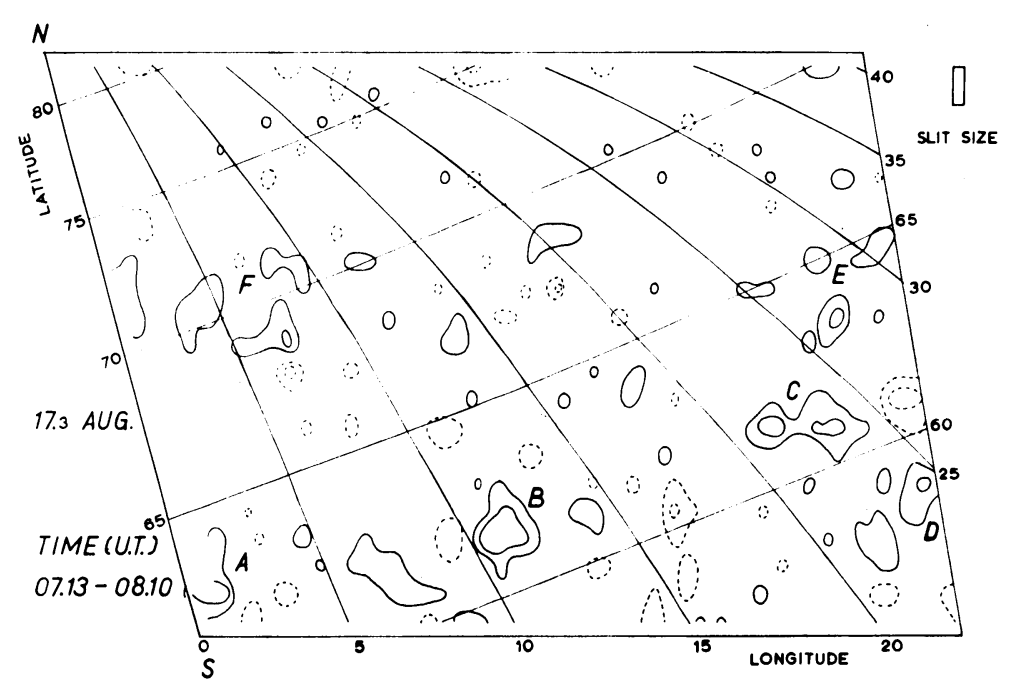

Fig. 4b.

Fig. 4a-b. Isogauss maps for the north pole obtained on August 16 at Mt Wilson (a) and on August 17 at Crimea (b). Solid lines represent $\mathrm{N}$ polarity, dashed lines $\mathrm{S}$ polarity. The isogauss levels are 5, 10 and $20 \mathrm{G}$.

A more quantitative comparison is presented in Figure 5, in which the average radial field $\bar{H}_{r}=\overline{H_{\|} / \cos \theta}$, the average longitudinal $\mathrm{N}$ - and S-polarity fields, $\bar{H}_{\mathrm{N}}$ and $\bar{H}_{\mathrm{S}}$, and the percentage of the scanned area covered by $\mathrm{N}$-polarity fields, $100 N_{\mathrm{N}} / N$, are given as functions of latitude. $N_{\mathrm{N}}$ is the number of observation points with fields of $\mathrm{N}$ polarity, while $N$ is the total number of points. Corrections have been made for 
the asymmetric position and time difference of the Crimean recording relative to the Mt Wilson one, so that the values in Figure 5 refer to the same area on the Sun. Only the results from August 20 are shown in Figure 5; the results from August 17 are qualitatively the same.

Considering the many factors that may influence recordings of the polar fields, the agreement between the Crimean and Mt Wilson results can be regarded as satisfactory. The correlation coefficient between the Crimean and Mt Wilson values of $\bar{H}_{r}$ is 0.7 for the north and 0.5 for the south hemisphere. The conclusions on the latitude variation of the polar fields that were based on the Mt Wilson recordings are thus supported by the Crimean data.

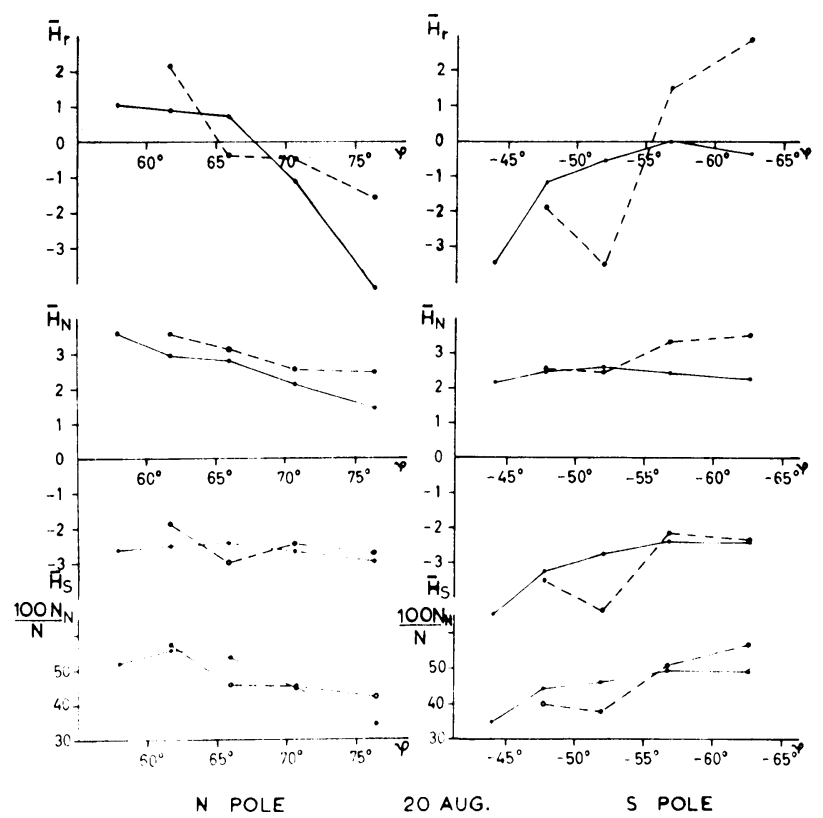

Fig. 5. The latitude variation of $H_{r}, H_{\mathrm{N}}, H_{\mathrm{S}}(\mathrm{G})$ and $100 N_{\mathrm{N}} / N$ as determined with the two magnetographs for August 20. Solid lines and filled circles: Mt Wilson. Dashed lines and open circles: Crimea.

\section{Remeasurements of Hale's Plates for the General Magnetic Field}

Hale's early results on the Sun's general magnetic field contradict the results of modern photoelectric observations. Practically all the plates used by Hale in the investigation of the general field were reduced by Van Maanen by visual settings with a tipping glass-plate micrometer.

To free the reductions from any possible personal bias, a few hundred of the plates from the 1914 series were chosen at random and taken by the author to the Sacramento Peak Observatory, where they could be automatically scanned with a digitized microphotometer. The line profiles were recorded on magnetic tape, and the reductions were made with a CD 3600 in Uppsala (Stenflo, 1970b). 
The same plates had been measured by Van Maanen. His reduction book with all his micrometer settings was available. This material had then been used for the determination of the inclination and period of rotation of the magnetic pole relative to the Sun's rotational axis.

Each plate is composed of $2 \mathrm{~mm}$ wide strips caused by the compound $\lambda / 4$ plate. Successive strips are alternately right-: and left-handed circularly polarized. On each plate there is a 'marked strip'. The adjoining strips are circularly polarized in the left-handed direction.

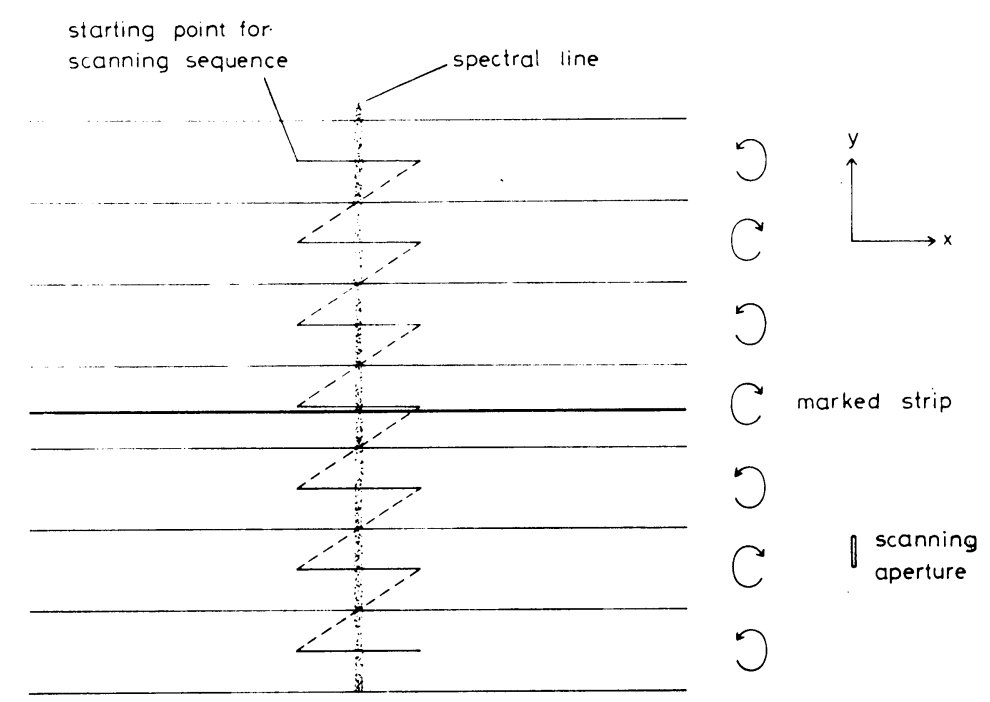

Fig. 6. Illustration of the procedure for automatic scanning of the Hale plates.

The scanning sequence is illustrated in Figure 6 . The position (mean $x$-coordinate) of the line in each strip was computed. Strips with defects (specks in the emulsion etc.) were skipped. The marked strip was always skipped in the computations. In Figure 7 are illustrated the line displacements $\bar{X}_{k}$ relative to strip no. 1. A least-mean-square fit of a second-order curve has been made to the values for strips of odd ordinal number, i.e. strips of the same polarization as strip no. 1 (filled circles in Figure 7). The displacement caused by a magnetic field is defined as the difference between the $\bar{X}_{k}$ in strips of even ordinal number (open circles in Figure 7) and the curve. The correct sign of the Zeeman displacement depends on (1) if the marked strip and strip no. 1 are of the same or opposite polarization, and (2) if the compound $\lambda / 4$ plate was in the normal or inverted position.

The average line displacements are given in Figure 8 as a function of heliographic latitude. The same spectral lines as those used by Van Maanen, the CrI lines $\lambda \lambda$ 5247.57, 5300.75 and $5329.15 \AA$ were used. The results obtained for each line separately are very similar. Accordingly only the results for all three lines together are given in Figure 8. Using the effective Landé factors of the lines we can express the displace- 
ments in $\mathrm{G}$. The scale to the right in Figure 8 , in which $1 \mu$ corresponds to $4 \mathrm{G}$, represents an average for the three lines.

Van Maanen's results for the same plates as were remeasured are shown in Figure 8a. The measured field shows a neat variation with heliographic latitude, with the solar equator forming a sharp demarcation line between the opposite polarities in the two hemispheres. The field strength attains a maximum of about $11 \mathrm{G}$ at lat. + and $-45^{\circ}$.

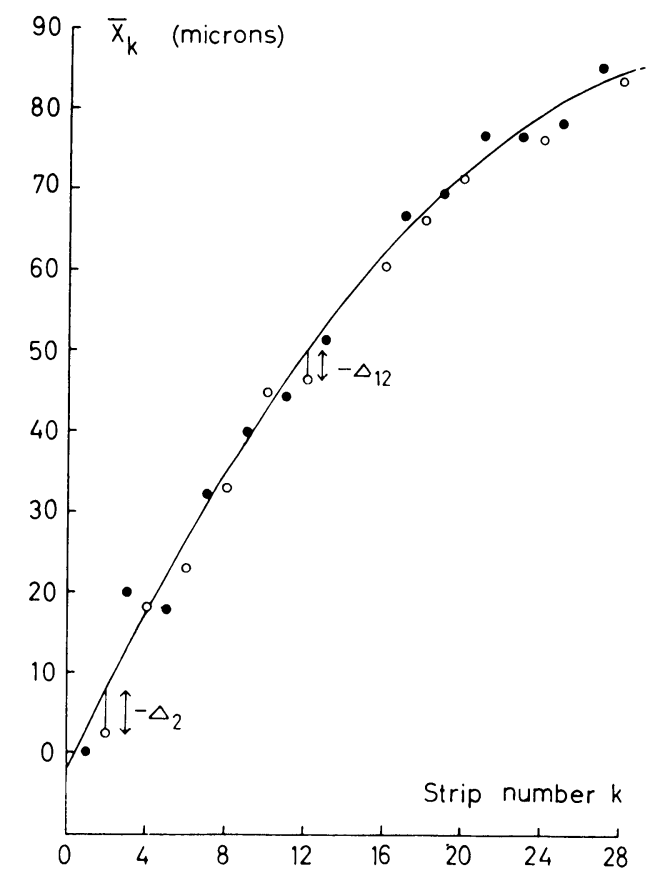

Fig. 7. Displacement $\bar{X}_{k}$ of the line in strip No. $k$ relative to the line in strip No. 1 . The filled circles represent values in strips of odd numbers (of the same polarization as strip No. 1), while the open circles represent values in strips of even numbers. The curve represents a second-order least-meansquare fit to the filled circles. The Zeeman displacement $\Delta_{k}$ is defined as the distance (with sign) of an open circle from the curve as illustrated in the figure.

In contrast, the computer reductions do not show any significant field at any latitude. It was not clear if north was 'up' or 'down' on the plates, and therefore two separate reductions were made to cover both these cases. Figure $8 \mathrm{~b}$ gives the results for the assumption that north was in the direction of decreasing strip numbers, while Figure $8 \mathrm{c}$ is based on the opposite assumption. An approximate upper limit for the average field strength is $5 \mathrm{G}$ for all latitudes. There is no correlation between the remeasured displacements and Van Maanen's values.

The standard deviations in the mean values are also shown in Figure 8. Van Maanen's standard deviations are about 3 times smaller than ours. His standard deviations in one value are only 1-2 $\mu$, which is quite remarkable, considering that the width of the spectral line was about $500 \mu$, that the exposure time was about $20 \mathrm{~min}$ and that the 
standard deviations should include the influence of local magnetic fields and Doppler shifts that are not coherent over successive strips. Our standard deviations in one value are $3-6 \mu$.

It is interesting to compare the attempts to determine the Sun's general magnetic field with the determinations of proper motions in the spiral galaxy M33. Van Maanen found the proper motions to be directed along the spiral arms (Lundmark, 1927).
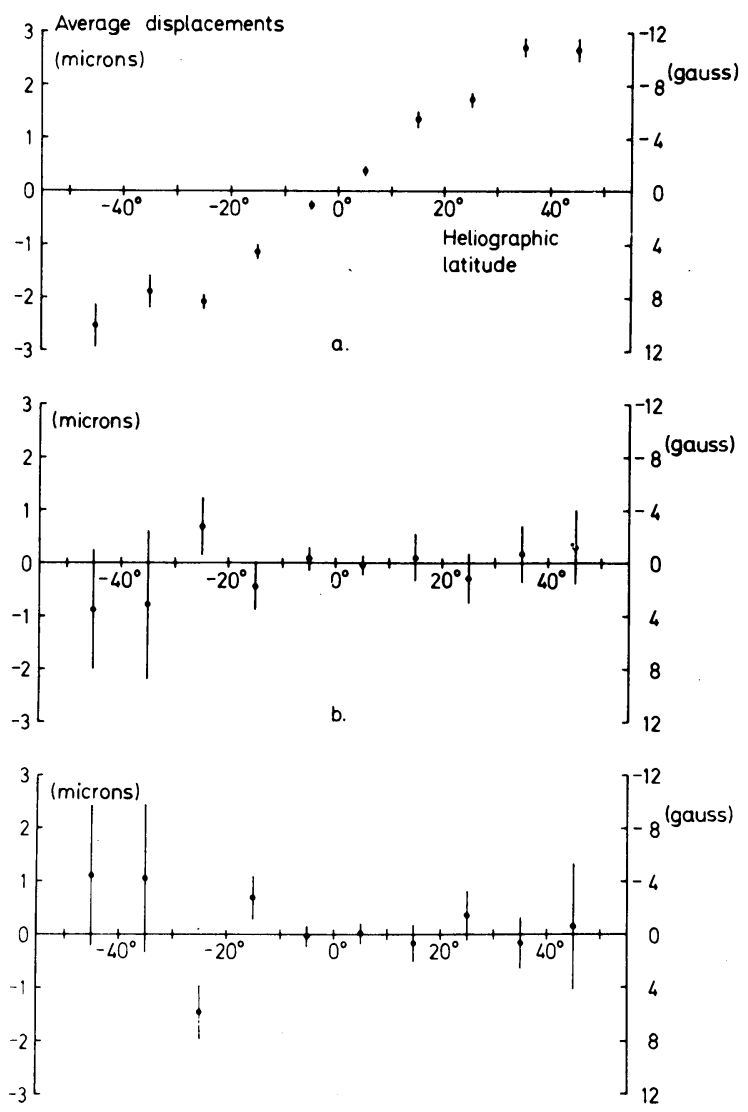

Fig. 8. Average Zeeman displacements expressed in microns and gauss as a function of heliographic latitude. The material has been grouped in the latitude intervals $-50^{\circ}--40^{\circ},-40^{\circ}--30^{\circ}, \ldots$, $+40^{\circ}-+50^{\circ}$. The standard deviations in the mean value are shown. (a) Van Maanen's results; (b) New results with the assumption that north is in the direction of decreasing strip numbers; (c) New results, with the assumption that north is in the direction of increasing strip numbers.

The proper motions were largest at the edge of M33 and decreased towards the centre of the galaxy, where they reversed direction. The rotation period found by Van Maanen was $230000 \mathrm{yr}$, which is smaller by a factor of 1000 than the period of rotation of our own galaxy. At that time these results were strong evidence against the idea that the so-called spiral nebulae were 'island universes' at large distances from our Milky Way system (Lundmark, 1927). 
According to our remeasurements of Hale's plates from 1914 there is no contradiction between the field observed at that time and the magnetic field that is presently observed with photoelectric techniques.

\section{References}

Babcock, H. D.: 1959, Astrophys. J. 130, 364.

Babcock, H. W.: 1961, Astrophys. J. 133, 572.

Babcock, H. W. and Babcock, H. D.: 1955, Astrophys. J. 121, 349.

Bumba, V., Kleczek, J., Olmr, J., and Ruzickova-Topolova, B.: 1969, Bull. Astron. Inst. Czech. $20,67$.

Hale, G. E.: 1912, Terrest. Magn. Atmospheric Electr. 17, 173.

Hale, G. E.: 1913, Astrophys. J. 38, 27.

Hale, G. E., Seares, F. H., Van Maanen, A., and Ellerman, F.: 1918, Astrophys. J. 47, 206.

Harvey, J. and Livingston, W.: 1969, Solar Phys. 10, 283.

Kotov, V. A. and Stenflo, J. O.: 1970, Solar Phys. 15, 265.

Leighton, R. B.: 1969, Astrophys. J. 156, 1.

Lundmark, K.: 1927, Uppsala Astron. Obs. Medd. No. 30.

Rosseland, S.: 1925, Astrophys. J. 62, 387.

Seares, F. H., Van Maanen, A., and Ellerman, F.: 1918, Proc. Nat. Acad. Sci. 4, 4.

Seares, F. H., Van Maanen, A., and Ellerman, F.: 1919, Proc. Nat. Acad. Sci. 5, 242.

Steenbeck, M.: 1966a, Wissenschaft und Menschheit 2, 315, Urania-Verlag Leipzig/Jena/Berlin.

Steenbeck, M. and Krause, F.: 1966b, Z. Naturforsch. 21a, 1285.

Steenbeck, M. and Krause, F.: 1967, Gustav-Hertz-Festschrift, Akademie-Verlag, Berlin.

Steenbeck, M. and Krause, F.: 1969, Astron. Nachr. 291, 49.

Stenflo, J. O.: 1970a, Solar Phys. 13, 42.

Stenflo, J. O.: 1970b, Solar Phys. 14, 263.

Waldmeier, M.: 1960, Z. Astrophys. 49, 176.

Waldmeier, M.: 1969, Astron. Mitt. Zürich Nr. 288.

\section{Discussion}

Livingston: Did you compare or try to correlate your Mt Wilson polar fields with white light polar faculae or other brightness pic tures? I am thinking that if no facular correspondence is seen the fields might be diffusely weak and $\mathbf{5 2 5 0}$ might not be thermally weakened (so your field strength as shown might be correct).

Stenflo: I have not compared my magnetic-field observations with brightness pictures.

Rust: A look at your last slide makes me think that a least-squares fit to your reduction of the data would imply polar fields of the same sign, if not of the same magnitude, as in the earlier reduction. A slide that Prof. Severny just showed seemed to include this point, and it showed that the polarity of the Sun's dipole was opposite to that to be expected from extrapolation of modern observations back to that time. Does your conclusion agree with this?

Stenflo: The conclusion I can draw from my reductions is that there is no significant field in excess of $5 \mathrm{G}$ at any latitude. It is not possible to determine the average polarity of the field from my data. 